\title{
Krankenversicherung der KPT
}

\section{Profitieren Sie vom FMH Insurance Services Kollektivvertrag}

\section{Ausgezeichnetes Preis-Leistungs-Verhältnis}

Treten Sie dem FMH Insurance Services Kollektivvertrag bei, welcher mit der KPT abgeschlossen wurde. Profitieren Sie von den Topleistungen und den attraktiven Prämienrabatten.

\section{Ihr Profit}

Auf folgenden Zusatzversicherungen offerieren wir Ihnen eine Vergünstigung:

\section{Spitalkosten $-15 \%$ Rabatt}

Übernahme von Spitalkosten, wahlweise auf der allgemeinen, halbprivaten oder privaten Abteilung.

\section{Krankenpflege-Plus - $10 \%$ Rabatt}

Erweiterte Kostenübernahme bei ambulanten medizinischen Leistungen wie Medikamenten, Brillen, Vorsorgeuntersuchungen, 8 Wochen Reiseversicherung, Impfungen usw.

\section{Natura -10\% Rabatt}

Kostenübernahme für alternative Heilmethoden bei Ärzten sowie anerkannten Therapeuten.

Darüber hinaus bieten wir attraktive Prämien für die obligatorische Krankenpflegeversicherung.

Dieses Angebot gilt für sämtliche FMH-Mitglieder und im gleichen Haushalt lebende Familienangehörige und Konkubinatspartner. Ein Beitritt ist bis zum 70. Altersjahr möglich.

Sind Sie bereits bei der KPT versichert? Rufen Sie uns an, damit Sie in Zukunft auch von günstigeren Konditionen profitieren können.

\section{Profitieren Sie von unserem Angebot}

Nutzen Sie die Vorteile des FMH Insurance Services Kollektivvertrags und bestellen Sie noch heute Ihre persönliche Offerte mit dem unten stehenden Antworttalon (bitte eine aktuelle Policenkopie beilegen).

\section{Antworttalon}

Vorname / Name

Adresse

PLZ / Ort

Geburtsdatum

Telefon Privat/Geschäft

Beste Zeit für einen Anruf

Bitte senden Sie mir eine Offerte. Eine aktuelle Policenkopie liegt bei.

Ich wünsche eine persönliche Beratung. Bitte rufen Sie mich an.

Ich interessiere mich für folgende Produkte:

\footnotetext{
Todesfallversicherung

Säule $3 a$

Pensionskasse BVG

Rechtsschutzversicherung

O Finanz-/Steuerplanung

Berufshaftpflichtversicherung
}

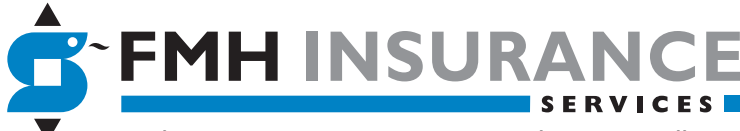

Roth Gygax \& Partner AG \& Koordinationsstelle Moosstrasse 2 — 3073 Gümligen

Telefon 0319595000 a Fax 0319595010 mail@fmhinsurance.ch $\square$ www.fmhinsurance.ch 